\title{
Towards Low-Cost Mems Imu Gait Analysis: Improvements Using Calibration and State Estimation
}

Lucas Samuel Lincoln, Eric Allen Johnson and Stacy J. Morris Bamberg

Biolnstrumentation Laboratory, University of Utah, USA

\begin{abstract}
Inexpensive, unobtrusive 3D motion tracking of human gait is of increasing interest for the medical and entertainment industries. Of particular interest are rehabilitative applications. For instance, being able to measure foot travel, e.g. stride length or foot clearance, would be very useful. Approaches using low-cost MEMS inertial measurement units have often been limited by requiring expensive calibration procedures and by the sensor's inherent noise and bias drift. The authors apply two techniques to improve IMU based gait tracking: a novel calibration routine and a zero-velocity bias update algorithm. The application of these aids reduces error by an average of $99.55 \%$ over six trails. Results show a $5.96 \%$ tracking accuracy in the progressive direction, which corresponds to errors on the centimeter scale.
\end{abstract}

\section{Introduction and Motivation}

Human motion tracking and analysis is an active subject of study covering a large range of applications from healthcare to entertainment. Traditionally, human motion tracking has relied on external camera or magnetic systems, obtrusive instrumentation, or high cost equipment; a review of techniques may be found in [1]. In a clinical sense, the former both have the ability to influence analysis thought patient physical or mental discomfort, and the latter can be inhibiting to largescale or long-term monitoring.

MEMS inertial measurement units (IMU), consisting of accelerometers and angular rate gyroscopes, have been applied to segment tracking for applications from the medical field to the entertainment industry [2-13]. IMUs have the benefit of being relatively small and unobtrusive, unlike instrumented linkages, and they are also self contained (requiring no specific environment for operation), unlike stereographic camera, vision, and magnetic tracking systems. Their mass adoption in the consumer electronics world has resulted in powerful sensors for continually decreasing cost and size. However, common problems exist in IMU use for segment tracking, predominantly the bias drift of the sensors and the non-linearities inherent in low-cost manufacturing, both of which notably introduce imperfect gravity cancellation [14].

Recent advances in real-time bias adjustment by the authors [15] as well as the development of a novel, inexpensive calibration system for low-cost IMUs [16], have encouraged a revisiting of the applicability of IMU gait analysis in general application, in particular with regard to accuracy and system cost.

The low-cost calibration of MEMS inertial sensors provides complex sensor models that account for scale factor nonlinearity, anisotropic sensitivity, and gyro specific force sensitivity [16].

This affords greater accuracy of measurement, particularly in estimating the required gravity cancellation. When double-integrating the acceleration signals for position even, small errors and unaccounted angular manufacturing tolerances can greatly affect the accuracy of tracking. This problem has typically limited the usefulness of IMU motion tracking for any real world tasks.

Our previous state estimation work [15] demonstrated that for human-scale, intermittent motion a sensor-level algorithm based on easily measured parameters enables real-time bias adjustment. This system is non-specific to application provided there are regular periods of rest. This has been demonstrated successfully on a small unmanned ground vehicle [16].

This paper investigates whether human gait provides long enough periods of rest to apply the state estimation algorithm for bias adjustment. This, combined with the calibration system developed, affords low-cost IMU based bioinstrumentation for human-motion tracking.

\section{Materials and Methods}

\section{Inertial measurement unit}

The IMU used is shown in Figure 1. Details of the sensor can be found in [16]. In short, the external dimensions are within $50 \times 37$ $\times 75 \mathrm{~mm}$ and it contains four independent accelerometer triads and two independent gyrotriads. The sensors are sampled at $1000 \mathrm{~Hz}$ with a 16-bit A/D. The redundancy of the sensors allows for a weighted combination based on the static variance of each individual axis. At any time, saturated sensors are weighted out of the resulting signal.

\section{Calibration}

The calibration system previously developed, fully described in [16], and provides an average improvement of 5.7\% and $24 \%$ over linear models for the accelerometers and gyros, respectively. This improves the state estimation described above, and combined they greatly reduce the compounding error resulting from multiple integrations over time. The calibration hardware is low cost and consists of a series of blocks manufactured to specific linear and angular dimensions (Figure 2). By exciting combinations of gyro- and accelerometer- axes through a predefined series of pure translational and rotational motions, a novel mathematical model is populated. This model includes non-

*Corresponding author: Stacy J. Morris Bamberg, Biolnstrumentation Laboratory, University of Utah, Salt Lake City, UT84101, E-mail: sjm.bamberg@utah.edu

Received November 06, 2011; Accepted November 21, 2011; Published November 22, 2011

Citation: Lincoln LS, Johnson EA, Bamberg SJM (2011) Toward Slow - Cost Mems Imu Gait Analysis: Improvements Using Calibration and State Estimation. J Bioengineer \& Biomedical Sci S1:006. doi:10.4172/2155-9538.S1-006

Copyright: (c) 2011 Chehade MJ, et al. This is an open-access article distributed under the terms of the Creative Commons Attribution License, which permits unrestricted use, distribution, and reproduction in any medium, provided the original author and source are credited. 
Citation: Lincoln LS, Johnson EA, Bamberg SJM (2011) Toward Slow - Cost Mems Imu Gait Analysis: Improvements Using Calibration and State Estimation. J Bioengineer \& Biomedical Sci S1:006. doi:10.4172/2155-9538.S1-006

linearities, anisotropic effects and gyro-acceleration sensitivity. The hardware is low-cost and the calibration straightforward; however 180 actions are required to define the model. This results in a calibration time of approximately 4-6 hours. For home or clinical healthcare, a 6 hour calibration at each use would severely limit the applicability and adoption of an IMU based motion tracking device. This paper investigates the temporal stability of IMU calibration, that is: will the device require recalibration at each use; and if not re-calibrated at each use, how much benefit is lost? It is proposed that if the calibration is relatively static then periodic human segment motion can be accurately captured using wearable IMUs that are calibrated prior to the first use.

\section{State Estimator}

The state estimator works as described in [16]. It is a method of zerovelocity update intended to be application inspecific and was originally

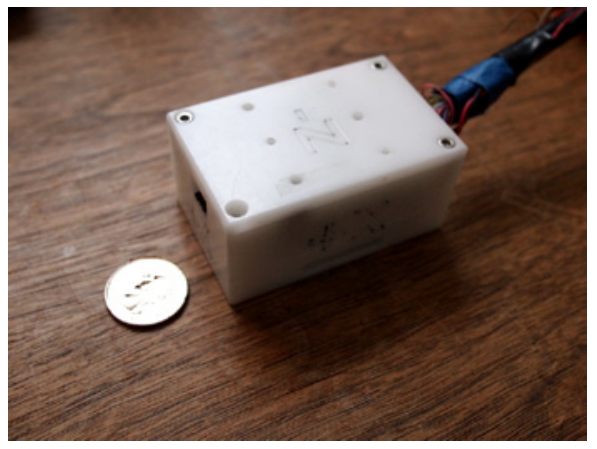

Figure 1: Photo of IMU, with US quarter for scale.

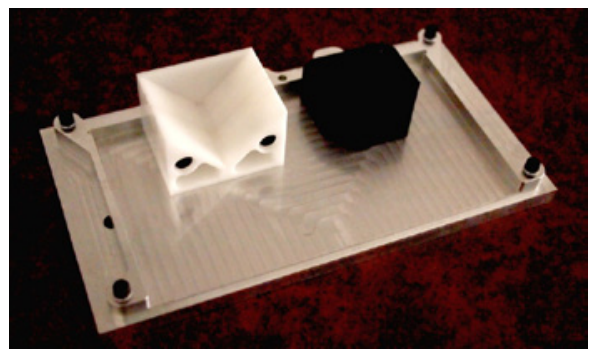

Figure 2: Photo of calibration hardware

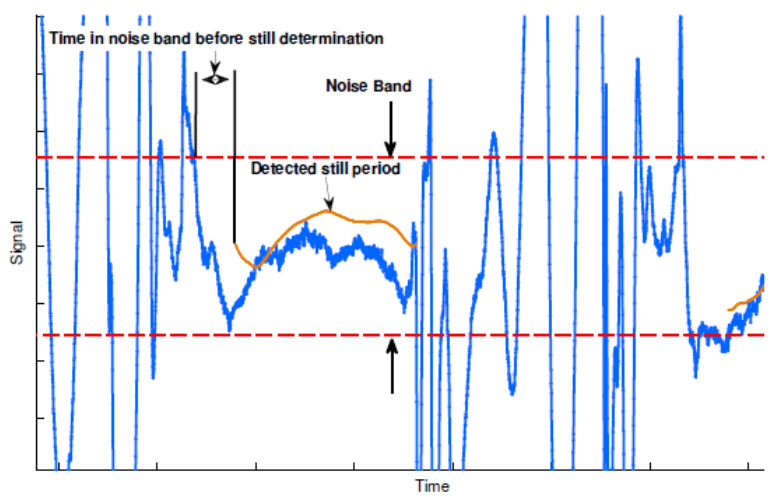

Figure 3: Still period detection on a representative trial showing tunable parameters of algorithm: Noise band and still period allowed prior to bias reset.

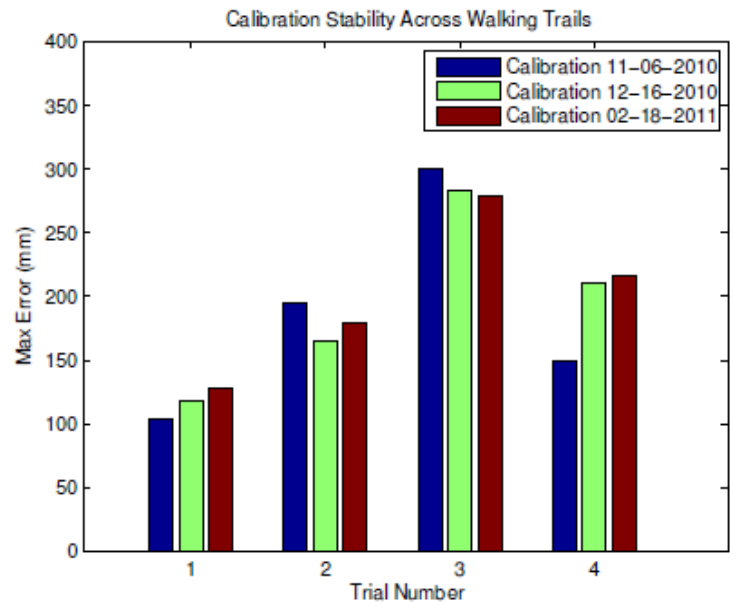

Figure 4: Graph showing relative stability of calibrations.

developed for mobile robotics tracking in GPS deficient environments. Zero velocity updates use a still period to re-bias accelerometer and gyros to minimize cumulating integration errors; they are typically based on step detection (using simple feature detection in accelerometer and gyro- scope signals $[17,18]$ or insole pressure measurement) and assume a no-slip condition with the ground. If a slip occurs, this error is accumulated for the remainder of the trial. The method described herein uses the measured noise parameters of the IMU to determine periods of still, therefore increasing the robustness of the system in irregular terrain and eliminating the no-slip requirement. The biasadjusting estimator has two tunable parameters for both accelerometers and gyroscopes: the length of time a signal must drop into the noise band of the sensor before being considered a still period (rather than noise or other abnormalities) and what magnitude to consider the noise band. These parameters are optimized on a given trial and applied to the algorithm prior to execution.

\section{Experimental Procedure}

Experiments were conducted in a motion analysis laboratory (MAL) which contains an 8-camera stereographic motion capture system (Vicon, Oxford, UK) for 3d motion tracking. The system has sub-millimeter accuracy and was utilized at a $1 \mathrm{kHz}$ capture rate. The IMU was attached to the rigid cap of a steel-toed boot. Mounted securely to the IMU was an L shaped block with vision-system markers identifying the IMU coordinate system. The IMU is attached via a shielded cable to the DAQ system and powered by a constant DC power supply. Two types of trials were performed: Normal, unaffected gait on level ground (four trials) and natural stair climbing and descent of three steps (two trials). Both types of trails were used to examine calibration stability and the contributions of each aid (calibration and bias-update routine).

\section{Analysis Methods and Techniques}

A goal of this work was to determine the usefulness of the two tools (calibration and bias-update) and as such each gait trail was analyzed to determine the maximum error between IMU measurement and ground truth in a six configurations:

- Bias-update Off, Factory Calibration 


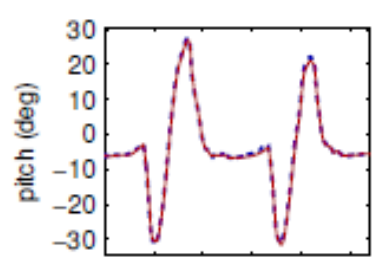

\section{--1MU}
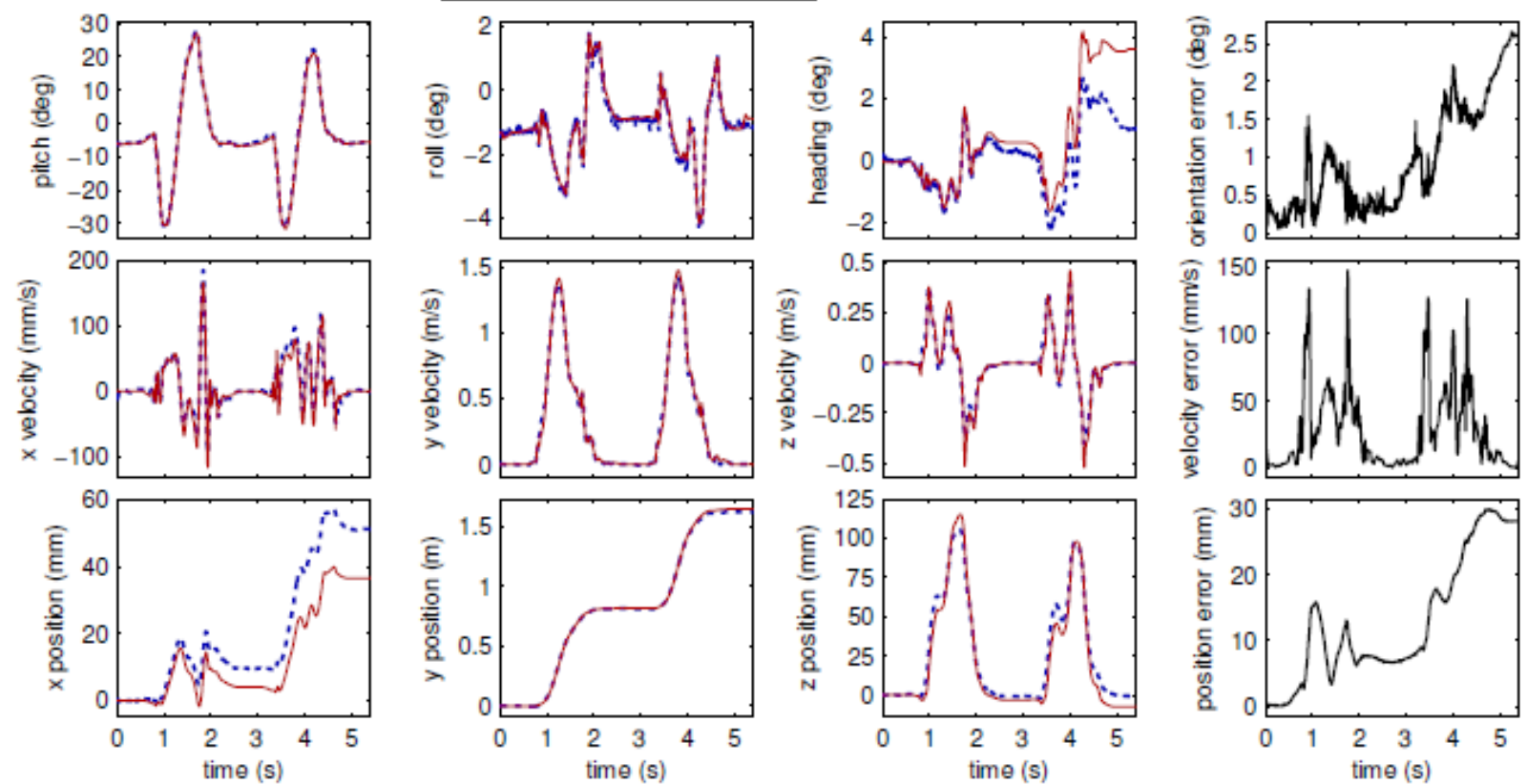

Figure 5: Plot showing IMU and camera tracking of the most accurate walking trial. Errors are in rightmost plots.
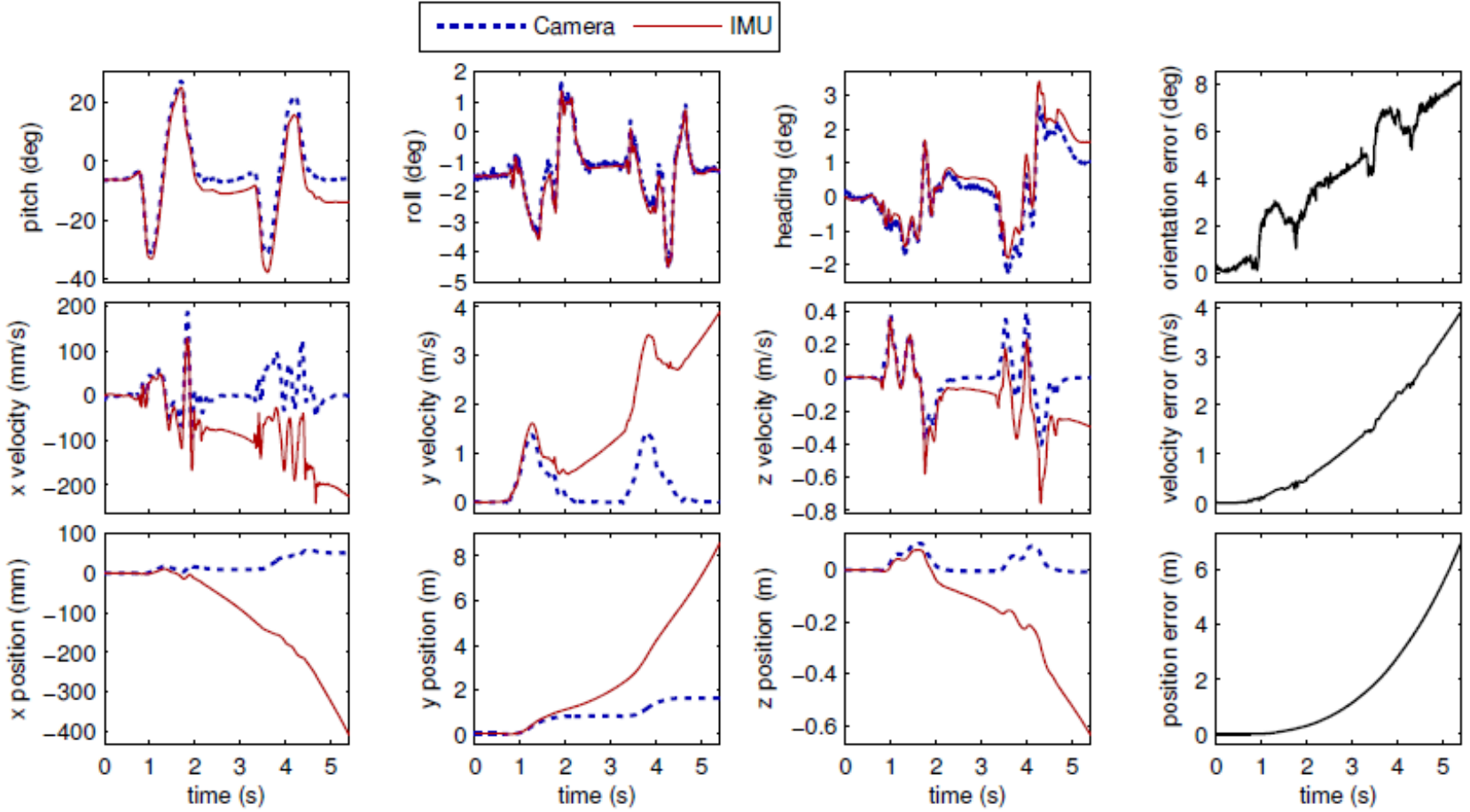

Figure 6: Plot showing same trial as Figure 5 with both calibration and state estimation aids removed. Note the rapidly compounding errors. 

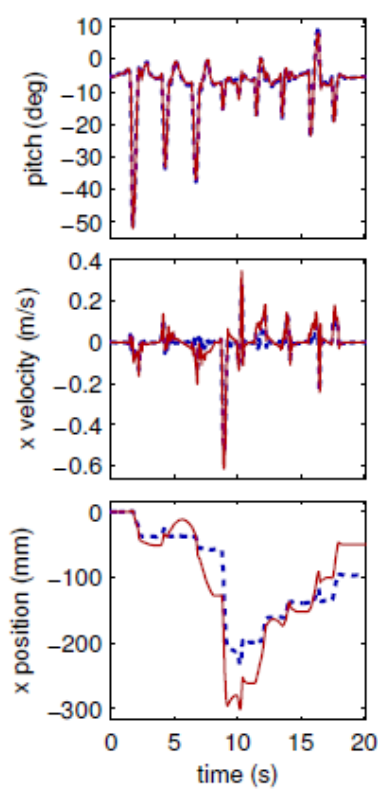
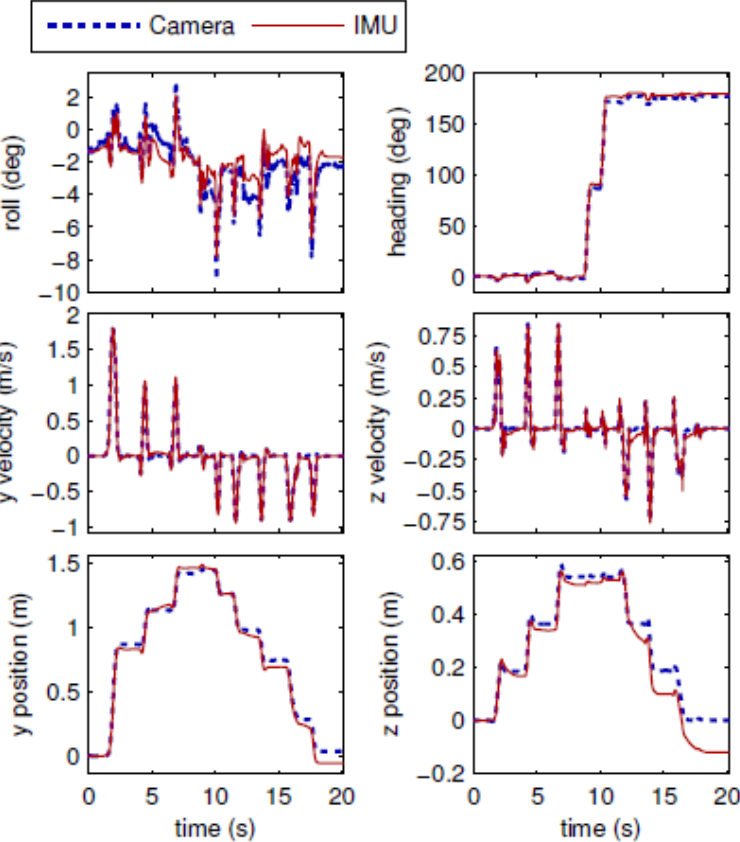
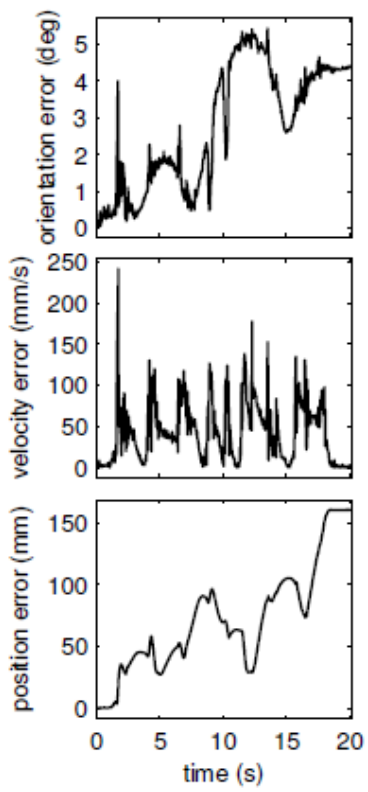

Figure 7: Plot showing IMU and camera tracking of a stairs trial. Errors are in right-most plots.

- Bias-update On, Factory Calibration

- Bias-update Off, User Calibration 1

- Bias-update On, User Calibration 1

- Bias-update On, User Calibration 2

- Bias-update On, User Calibration 3

This set of routines provides information as to the contribution of each tool; as well as providing an indication of the stability of calibrations by applying calibrations 1,2 and 3 to the trails under otherwise identical conditions. Note calibrations 1 and 2 are from a common operator and 3 a different operator.

\section{Results}

To investigate the stability of calibration, Figure 4 demonstrates the relative difference in error by using three calibrations from different dates on the four walking gait trials. Likewise, to determine the relative effect of bias- adjustment and calibration, Table 1 displays the percent error reduction by applying our calibration routine, applying the biasupdate algorithm; and applying both.

To determine the success of a low-cost IMU for gait tracking, all trials were analyzed with state estimation on and our calibration active. Figure 5 shows the angle, velocity, and position IMU tracking as well as truth from the camera system of a walking trial. Errors are shown in the rightmost plots. Figure 6 is the same trial as in 5 with both aids disabled, i.e. no bias updates and using the factory calibration. Figure 7 is a representative stairs trial. Table 2 contains the errors as a percentage of total distanced travelled, directionally for $\mathrm{X}, \mathrm{Y}, \mathrm{Z}$ and rotations. $\mathrm{Y}$ is the direction of primary progress in all trials, $\mathrm{Z}$ height, and $\mathrm{X}$ the lateral axis.

\section{Discussion and Conclusions}

The results are greatly improved by the process of calibrating and applying zero-velocity updates, as evidenced by comparing the representative stair (Figure 7) and gait (Figure 5) tracking results using the aids to the same walking trial without (Figure 6). The results Table 1 quantifies the improvement made by each aid. Note that state estimation alone makes the largest contribution to the error reduction; but also that the calibration improvements are non-trivial. The additional improvement of the calibration above state estimation alone is $104.75 \mathrm{~mm} \pm 163.82 \mathrm{~mm}$.

The low-cost, self calibration routine appears to be static across the walking trials as seen in Figure 4. The data does not show a trend for more recent calibrations to be more accurate, therefore a single, careful calibration to a new IMU is suspected to be sufficient for some months. Studies will continue with continuing calibration to further determine the stability of the parameters. Absolute errors as a percent of the distance travelled are shown in Table 2. Note that the mean error in the direction of progress is approximately $6 \%$, with a particularly poor result in stair trial 2 . The reason for this trial's poor performance has not been identified. The relatively larger error percentages in $\mathrm{X}$ and $\mathrm{Z}$ are in part due to the minimal amount of displacement in those directions. Their absolute errors are lower than those of the progressive direction.

\section{Angular tracking is consistently strong across trials.}

The work shown within demonstrates the vast error reduction in in-expensive IMU-based motion tracking made by using a lowcost, clinically feasible calibration routine as well as a bias-updating zero-velocity update algorithm. 3D tracking has been previously demonstrated to achieve errors under $1 \%[4,19]$, however this work uses more costly, and high-performance gyroscopes. A goal of this 
Citation: Lincoln LS, Johnson EA, Bamberg SJM (2011) Toward Slow - Cost Mems Imu Gait Analysis: Improvements Using Calibration and State Estimation. J Bioengineer \& Biomedical Sci S1:006. doi:10.4172/2155-9538.S1-006

Page 5 of 5

\begin{tabular}{c|ccc} 
Trial & Calibration & State Estimation & Both \\
\hline Stairs 1 & $32.92 \%$ & $99.92 \%$ & $99.95 \%$ \\
Stairs 2 & $56.84 \%$ & $99.57 \%$ & $99.60 \%$ \\
Walking 1 & $33.03 \%$ & $99.01 \%$ & $99.65 \%$ \\
Walking 2 & $82.35 \%$ & $97.51 \%$ & $99.23 \%$ \\
Walking 3 & $88.15 \%$ & $99.45 \%$ & $99.46 \%$ \\
Walking 4 & $75.83 \%$ & $99.29 \%$ & $99.43 \%$
\end{tabular}

Table 1: Aid effects, as percent reduction of the error with no state estimation and with factory calibration.

\begin{tabular}{c||ccc|ccc}
\multicolumn{1}{l||}{} & \multicolumn{3}{c|}{ Position Errors (\%) } & \multicolumn{3}{c}{ Angular Errors (\%) } \\
Trial & $\mathrm{X}$ & $\mathrm{Y}$ & $\mathrm{Z}$ & $\mathrm{X}$ & $\mathrm{Y}$ & $\mathrm{Z}$ \\
\hline Stairs 1 & 15.77 & 3.05 & 7.40 & 1.63 & 1.03 & 0.68 \\
Stairs 2 & 17.45 & 20.30 & 9.25 & 1.55 & 1.06 & 1.01 \\
Walking 1 & 12.44 & 1.47 & 2.88 & 5.34 & 0.63 & 0.72 \\
Walking 2 & 21.81 & 3.68 & 21.59 & 1.22 & 1.31 & 0.83 \\
Walking 3 & 27.20 & 3.74 & 10.90 & 3.72 & 1.18 & 0.48 \\
Walking 4 & 14.30 & 3.52 & 13.05 & 1.38 & 1.27 & 0.74 \\
\hline Mean & 18.16 & 5.96 & 10.85 & 2.47 & 1.08 & 0.74
\end{tabular}

Table 2: Errors as a percent of distance travelled.

work is to achieve reasonable results without requiring expensive equipment.

Future work will include larger scale studies of gait using IMUs and validation of the technique. Incorporation of the IMU into a removable insole and application of on-board digitization and wireless transfer will both improve the accuracy of the results and the ease of use. This will enable application for rehabilitation, for instance: to track distance traveled or to provide real-time feedback to improve gait and stride length. Rehabilitation to improve range of motion is also of interest.

\section{References}

1. Zhou H, Hu H (2008) Human motion tracking for rehabilitation-a survey, Biomed signal Process Control 3: 1-8.

2. E. Foxlin (1996) Inertial head-tracker sensor fusion by a complementary separate-bias Kalman filter. IEEE Virtual Reality Annual Int. Symposium (VRAIS'96) 185-194,267

3. E. Foxlin, M. Harrington, Y. Altshuler (1998) Miniature 6-DOF inertial system for tracking HMDs, in Proc. SPIE Conf. on Helmet- and Head- Mounted Displays III, vol. 3362, (Orlando, FL, USA) 214-228, Apr. 13-14.

4. Foxlin E (2005) Pedestrian tracking with shoe-mounted inertial sensors. IEEE Comput Graph Appl 25: 38-46.
5. Bamberg SJ, Benbasat AY, Scarborough DM, Krebs DE,Paradiso JA (2008) Gait analysis using a shoe-integrated wireless sensor system. IEEE Trans Inf Technol Biomed 12: 413-423.

6. Yang J, Choi ES, Chang W, Bang WC, Cho SJ, et al. (2004) A novel hand gesture input device based on inertial sensing technique. IEEE Industrial Electronics Society 30th Annual Conf. (IECON'04) 3: 2786-2791.

7. Zhu R, Zhou Z (2004) A real-time articulated human motion tracking using tri-axis inertial/magnetic sensors package, IEEE Trans Neural Syst Rehabil Eng12: 295-302.

8. Goodvin C, Park EJ, Huang K, Sakaki K (2006) Development of a real-time three-dimensional spinal motion measurement system for clinical practice, Med Biol Eng Comput 44: 1061-1075.

9. Luinge HJ, Veltink PH (2005) Measuring orientation of human body segments using miniature gyroscopes and accelerometers, Med Biol Eng Comput 43 273-282.

10. Lee JK, Park EJ (2009) Minimum-order kalmanfilterwith vector se- lector for accurate estimation of human body orientation. IEEE Trans- actions on Robotics 25: 1196-1201.

11. Lee JK, Park EJ (2009) A fast quaternion-based orientation optimizer via virtua rotation for human motion tracking, IEEE Trans Biomed Eng 56: 1574-1582.

12. Miller N, Jenkins OC, Kallmann M, Mataric MJ (2004) Motion capture from inertial sensing for untethered humanoid teleoperation.. 4th IEEE-RAS Int. Conf. on Humanoid Robots 2: 547-565.

13. Wang JS, Hsu YL, Liu JN (2010) An inertial-measurement-unit- based pen with a trajectory reconstruction algorithm and its applica- tions, IEEE Transactions on Industrial Electronics 57: 3508-3521.

14. Woodman OJ (2007) An introduction to inertial navigation, Tech. Rep. 696 University of Cambridge, Computer Laboratory, $15 \mathrm{JJ}$ Thomson Avenue, Cambridge CB3 OFD.

15. Johnson EA, Bamberg SJM, Minor MA (2008) A state estimator for rejecting noise and tracking bias in inertial sensors. IEEE Int. Conf. on Robotics and Automation (ICRA'08) 3256-3263.

16. Johnson EA (2011) Investigating inertial measurement for human-scale motion tracking. PhD thesis, University of Utah. Department of Mechanical Engineering.

17. Jimnez CPAR, Seco F, Guevara J (2009) A comparison of pedestrian deadreckoning algorithms using a low-cost memsimu, in 6th IEEE International Symposium on Intelligent Signal Processing 37-42.

18. Ojeda L, Borenstein J (2007) Non-GPS navigation for security personnel and emergency responders. Journal of Navigation.

19. Foxlin E, Wan S (2010) Improved pedestrian navigation based on driftreduced MEMS IMU chip, in ION International Technical Meeting. InterSense Incorporated. 